\title{
On MIMO Channel Capacity, Correlations, and Keyholes: Analysis of Degenerate Channels
}

\author{
Sergey Loyka, Member, IEEE, and Ammar Kouki, Senior Member, IEEE
}

\begin{abstract}
It has recently been demonstrated that zero correlation of a random multiple-input multiple-output channel is not a guarantee of its high capacity. Degenerate channels exist, which have zero correlation and still low capacity. In this letter, we provide a statistical analysis of this phenomenon, formulate the general condition for a channel to be degenerate, and propose a method to estimate its capacity.
\end{abstract}

Index Terms-Channel capacity, correlation, keyhole, multipleinput multiple-output (MIMO).

\section{INTRODUCTION}

C HANNEL capacity of multiple-input multiple-output (MIMO) communication architecture is usually thought of as limited by correlation. It is low for highly correlated channels and it is high when the correlation between individual subchannels (i.e., links between one transmit and one receive antenna) of the matrix (MIMO) channel is zero. However, an elegant example has been presented in [1], which demonstrates that zero correlation is not a guarantee of high capacity, i.e., the channel may have zero correlation and still only a single degree of freedom. These are so-called degenerate channels or keyholes.

In this letter, we provide a statistical explanation of this phenomenon and, in particular, we emphasize that one should distinguish between "instantaneous" and "mean" (or conventional) correlation. We also present a general statistical criterion for the channel to be degenerate and propose a method to estimate the capacity of such channels.

\section{KeYHOLE}

An elegant example of a $2 \times 2$ channel, which has zero correlation and still only one degree of freedom (i.e., nonzero eigenvalue of $r_{i j}$, see (2), or nonzero singular value of $\mathbf{H}$ ), has been presented in [1]. A brief description of this example is given below for completeness. Consider a $2 \times 2$ MIMO system in a rich multipath environment where all the components of the matrix channel are uncorrelated complex Gaussian variables. This channel has two degrees of freedom and, consequently, high capacity, because by using appropriate signal processing at the receiver, it can be transformed into two "virtual" parallel inde-

Paper approved by N. C. Beaulieu, the Editor for Wireless Communication Theory of the IEEE Communications Society. Manuscript received April 19, 2001; revised March 9, 2002

S. Loyka is with the School of Information Technology and Engineering (SITE), University of Ottawa, Ottawa, ON K1N 6N5, Canada (e-mail sergey.loyka@ieee.org).

A. Kouki is with the Department of Electrical Engineering, Ecole de Technologie Superieure, Montreal, QC H3C 1K3, Canada.

Digital Object Identifier 10.1109/TCOMM.2002.806543

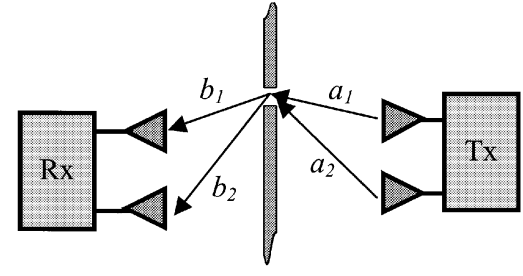

Fig. 1. Example of a degenerate $2 \times 2$ channel with a keyhole. $a_{1}, a_{2}, b_{1}$, and $b_{2}$ independent complex Gaussian variables.

pendent channels. Now let us place a screen with a small hole (keyhole) between transmit and receive arrays (see Fig. 1). The signal radiated by the transmit array can propagate to the receive array only through the keyhole. The channel matrix in this case takes the following form [1]:

$$
\mathbf{H}=\left(\begin{array}{l}
b_{1} \\
b_{2}
\end{array}\right) \sigma\left(\begin{array}{ll}
a_{1} & a_{2}
\end{array}\right)=\sigma\left(\begin{array}{ll}
a_{1} b_{1} & a_{2} b_{1} \\
a_{1} b_{2} & a_{2} b_{2}
\end{array}\right)
$$

where $\sigma$ is the scattering cross-section of the keyhole, $a_{1}$ and $a_{2}$ are the channel coefficients from the transmit array to the keyhole, and $b_{1}$ and $b_{2}$ are the channel coefficients from the keyhole to the receive array. All coefficients are independent complex Gaussian variables. Clearly, the entries of $\mathbf{H}$ are uncorrelated. However, this channel has only one nonzero singular value (by construction) and, hence, there is only one degree of freedom, meaning that the channel capacity is low. Such a channel may be referred to as a degenerate channel. Some more realistic examples of such channels have also been discussed in [1].

\section{CAPACITY OF RANDOM (STOCHASTIC) MIMO ChanNEL}

The capacity of a fixed linear $n \times n$ matrix channel with additive white Gaussian noise (AWGN) and when the transmitted signal vector is composed of statistically independent equal power components, each with a Gaussian distribution, and when the receiver knows the channel, may be presented in the following form [2], [3]:

$$
C=\log _{2} \operatorname{det}\left[\delta_{i j}+\frac{\rho}{n} \cdot r_{i j}\right] \text { bits/s/Hz }
$$

where $n$ is the number of transmit/receive antennas, $\rho$ is the signal-to-noise ratio (SNR), $\delta_{i j}$ is $n \times n$ identity matrix, $r_{i j}$ are the entries of the channel correlation matrix

$$
r_{i j}=\sum_{k} h_{i k} h_{j k}^{*}
$$

$h_{i j}$ is the normalized channel matrix (frequency independent over the signal bandwidth), i.e., the normalized transfer factor from $j$ th transmitter to the $i$ th receiver, and ${ }^{*}$ denotes complex conjugate. When the channel is random, the correlation matrix 
and the capacity are random as well. In this case, we call $r_{i j}$ "instantaneous" correlation (i.e., for a given channel realization) to distinguish it from conventional or "mean" correlation (with the expectation over the channel matrix), and $C$ is "instantaneous" capacity (i.e., the capacity of a given channel realization). Further, the distribution function of $C$ may be determined [2], or, alternatively, the mean (ergodic) capacity may be considered [4]

$$
\langle C\rangle=\left\langle\log _{2} \operatorname{det}\left[\delta_{i j}+\frac{\rho}{n} \cdot r_{i j}\right]\right\rangle
$$

where \langle\rangle is the expectation over the channel matrix. Using Jensen's inequality and concavity of log det function [5], one obtains the following upper bound on $\langle C\rangle$ :

$$
\langle C\rangle \leq \bar{C}=\log _{2} \operatorname{det}\left[\delta_{i j}+\frac{\rho}{n} \cdot \overline{r_{i j}}\right]
$$

where $\overline{r_{i j}}$ is "mean" (conventional) correlation of the receive branches

$$
\overline{r_{i j}}=\sum_{k}\left\langle h_{i k} h_{j k}^{*}\right\rangle .
$$

Thus, the mean (or conventional) correlation provides the upper bound on capacity and says nothing about its mean or instantaneous values. This upper bound is not necessarily close to actual capacity and, as a detailed analysis shows [6], may be far away from the mean capacity in many cases.

\section{CAPACITY OF $2 \times 2$ MIMO CHANNEL}

Consider the specific case of a $2 \times 2$ MIMO channel. In this case, (2) takes the following explicit form:

$$
C=\log _{2}\left(1+\frac{\rho}{2}\left(r_{11}+r_{22}\right)+\left(\frac{\rho}{2}\right)^{2} r_{11} r_{22}\left(1-\left|R_{12}\right|^{2}\right)\right)
$$

where $R_{12}$ is the normalized correlation coefficient $\left(\left|R_{12}\right| \leq 1\right)$

$$
R_{12}=\frac{r_{12}}{\sqrt{r_{11} r_{22}}} \text {. }
$$

In fact, $r_{11}$ and $r_{22}$ represent the normalized received power in the first and second branches, respectively, and do not affect the number of nonzero eigenvalues (as we show below). The eigenvalues $\lambda$ of $r_{i j}$ can be obtained from the following equation:

$$
\lambda^{2}-\left(r_{11}+r_{22}\right) \lambda+r_{11} r_{22}\left(1-\left|R_{12}\right|^{2}\right)=0 .
$$

The singular values of $h_{i j}$ are the square roots of $\lambda$ [4]. Thus, correlation has the major impact on the number of degrees of freedom (i.e., nonzero singular or eigenvalues): there are two degrees of freedom when $\left|R_{12}\right|<1$, and only one when $\left|R_{12}\right|=$ 1 (as long as the received powers are not zero). Note that the number of degrees of freedom does not depend on $r_{11}$ and $r_{12}$.

When the channel is random, the mean (ergodic) capacity may be defined using the expectation over the channel matrix in (7) [see (4)] [4]. A detailed analysis using Monte-Carlo simulations of a correlated Rayleigh channel and of the channel in [1] shows that the impact of $r_{11}$ and $r_{22}$ on the mean capacity is much smaller than that of $\left|R_{12}\right|$ (the same conclusion may be obtained using Jensen's inequality). For example, Fig. 2 shows the rigorously computed mean capacity (i.e., using expectation

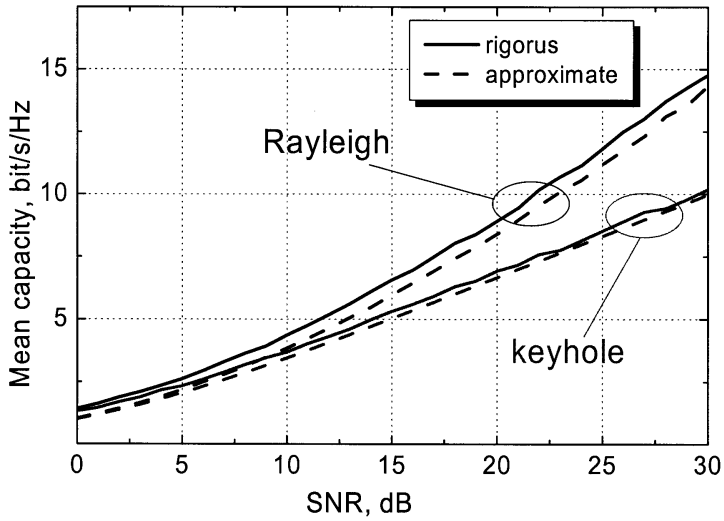

Fig. 2. Mean capacity of the correlated Rayleigh channel and of the keyhole channel in [1] versus $\operatorname{SNR}(\rho)$.

of (7) over channel realizations) and its approximate value, assuming $r_{11}=r_{22}=1$, of the correlated Rayleigh channel (with normalized correlation coefficient equal to 0.8 ) and of the keyhole channel in [1]. Obviously, $r_{11}$ and $r_{22}$ have a small effect on the mean capacity. This conclusion agrees well with the eigenvalue analysis above. Thus, to separate the effect of correlation from the effect of per-branch SNR and to study it in the explicit form, we further assume that $r_{11}=r_{22}=1$. In this case, the mean capacity depends on $R_{12}$ only, which is "instantaneous" correlation coefficient and can be presented as follows:

$$
\langle C\rangle=\int_{D R_{12}} C\left(R_{12}\right) \cdot f\left(R_{12}\right) d R_{12}
$$

where $f\left(R_{12}\right)$ is the probability density function (pdf) of $R_{12}$ and $D R_{12}$ is the range of $R_{12}$. Thus, the mean capacity depends on the pdf of $R_{12}$, not only on its mean value. In general, the mean correlation is not a reliable tool in estimating the MIMO capacity of a random channel. Let us now consider an illustrative example, when $R_{12}= \pm 1$ with equal probability. Obviously, the mean correlation is zero, but the mean capacity is low and there is only one degree of freedom just because $\left|R_{12}\right|=1$. The next example is the one presented in Section II. In this case

$$
R_{12}=e^{j\left(\varphi_{1}-\varphi_{2}\right)}
$$

where $\varphi_{1}=\arg \left(b_{1}\right), \varphi_{2}=\arg \left(b_{2}\right)$. Again, $\left\langle R_{12}\right\rangle=0$ because $\varphi_{1}$ and $\varphi_{2}$ are independent and uniformly distributed, but the mean capacity is low and there is only one degree of freedom because $\left|R_{12}\right|=1$.

From the considerations above, we may conclude the following.

- The capacity of deterministic channels is maximum when $R_{12}=0$ [see (7)]. However, as the examples above show, it is wrong to state the same about random channels using the mean correlation, i.e., zero mean correlation of a random channel is not a sufficient condition of maximum mean capacity. Using inequality (5), we conclude that it is the necessary condition (i.e., if the mean correlation is high, then the mean capacity is necessarily low).

- Referring to (7), we conclude that the sufficient condition to achieve high capacity is low-mean magnitude correlation. For example, if $\left\langle\left|R_{12}\right|\right\rangle=0$, then there are two 


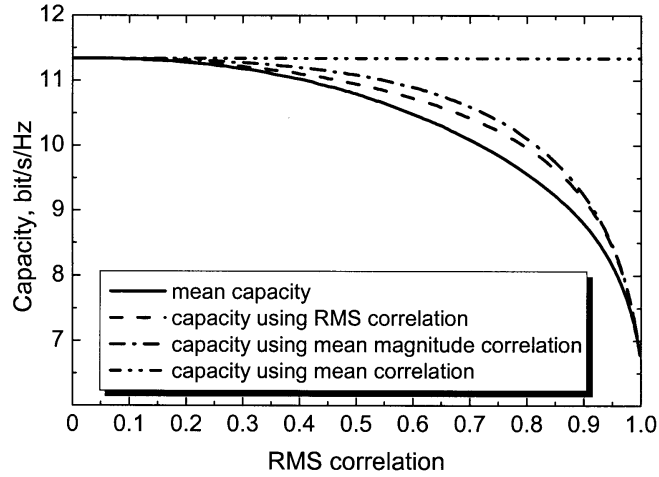

Fig. 3. MIMO capacity using root mean square (RMS), mean magnitude, and mean correlation versus RMS correlation for $\rho=20 \mathrm{~dB}$.

degrees of freedom and the mean capacity is maximum simply because $R_{12}=0$ in this case.

The general conditions for a channel to be degenerate are

$$
\left\langle R_{12}\right\rangle=0 \text { and }\left|R_{12}\right|=1 .
$$

From a practical viewpoint, $\left|R_{12}\right|$ may not be equal to one but be close to it. The capacity will be low in this case as well. In particular, it will be low when $\left|R_{12}\right| \geq 0.5-0.8$ [3], [8]. In the case of degenerate channels, the mean correlation does not provide an accurate estimation of the capacity. Fig. 3 illustrates the channel capacity for pdf of $R_{12}$ of the following form:

$$
f\left(R_{12}\right)=c \cdot \exp \left(\frac{\left|R_{12}\right|}{\alpha}\right), \quad\left|R_{12}\right| \leq 1
$$

where $c$ is normalizing constant and $\alpha$ determines the root mean square (RMS) value of $R_{12}$ (note that $\alpha$ may be negative as well as positive). All the curves in Fig. 3 were generated using (7) with $r_{11}=r_{22}=1$. Mean capacity curve was obtained by averaging (7) with respect to (13). Capacity using an RMS correlation curve and capacity using a mean magnitude correlation curve were obtained using (7) with $\left|R_{12}\right|$ set to be equal to $\sqrt{\left\langle R_{12}^{2}\right\rangle}$ and $\left\langle\left|R_{12}\right|\right\rangle$, correspondingly. Capacity using a mean correlation curve was obtained in a similar way. Obviously, the mean correlation is zero and its use for estimating the capacity will give an incorrect result. As Fig. 3 indicates, a more accurate estimation of the capacity of degenerate channels can be obtained using RMS or mean magnitude correlation. The results in [8] suggest that the similar conclusions should hold for $n>2$, as well.

\section{REFERENCES}

[1] D. Chizhik, G. J. Foschini, and R. A. Valenzuela, "Capacities of multi-element transmit and receive antennas: Correlations and keyholes," Electron. Lett., vol. 36, no. 13, pp. 1099-1100, June 2000.

[2] G. J. Foschini and M. J. Gans, "On limits of wireless communications in a fading environment when using multiple antennas," Wireless Personal Commun., vol. 6, no. 3, pp. 311-335, Mar. 1998.

[3] S. L. Loyka and J. R. Mosig, "Channel capacity of N-antenna BLAST architecture," Electron. Lett., vol. 36, no. 7, pp. 660-661, Mar. 2000.

[4] I. E. Telatar, "Capacity of multiantenna Gaussian channels," AT\&T Bell Lab. Internal Tech. Memo., 1995.
[5] T. M. Cover and J. A. Thomas, Elements of Information Theory. New York: Wiley, 1991.

[6] S. Loyka and A. Kouki, "On the use of Jensen's inequality for MIMO channel capacity estimation," in Proc. Canadian Conf. Electrical and Computer Engineering (CCECE 2001), Toronto, ON, Canada, May 13-16, 2001.

[7] _ - "New compound upper bound on MIMO channel capacity," IEEE Commun. Lett., vol. 6, pp. 96-98, Mar. 2002.

[8] S. L. Loyka, "Channel capacity of MIMO architecture using the exponential correlation matrix," IEEE Commun. Lett., vol. 5, pp. 369-371, Sept. 2001. 